\title{
Tuneable laser-based calibration and characterisation of radiometric detectors for SI-traceable measurements
}

Schuster, Michaela, Nevas, Saulius and Sperling, Armin

Physikalisch-Technische Bundesanstalt

Bundesallee 100, 38116 Braunschweig

\section{Introduction}

SI-traceable values of the spectral responsivity of radiometric detectors (generated photocurrent per unit of radiometric quantity) are of the highest importance in many applications in photometry and radiometry. For this evaluation, spectral responsivity measurements are carried out under exposure to monochromatic radiation. The electrical response of the device under test (DUT) is compared to that of a reference detector. The responsivity of the reference detector is known from a calibration with respect to the absolute cryogenic radiometer, an electrical substitution radiometer used as a primary standard for radiant power. These measurements are typically carried out in monochromator-based setups, where spectrally dispersed radiation of quartz-tungsten-halogen or gas-discharge arc lamps is normally used [1]. However, due to the rapid progress in the solid-state laser technologies, lamp-based setups can nowadays be replaced by tuneable laser facilities. The use of tuneable laser radiation has a significant advantages as compared to the classical lamp-monochromator setups. Much higher spectral output power levels and the simultaneously narrow bandwidth of the laser radiation during the measurements enable the e.g. DUT irradiance responsivity to be determined over a much broader dynamic range. In some cases, like the spectral calibration of industrial UV meters, tuneable lasers provide the nearly only direct possibility for a traceable calibration, because no lamp-monochromator setup is able to provide such high spectral irradiance levels equivalent to those met in industrial applications. Furthermore, steep slopes of the responsivity function of filtered detectors can be measured with much more detail using narrow-band lasers and, furthermore, no spectral deconvolution techniques (bandpass correction) are required as opposed to the monochromator-based measurements. Moreover, the wavelength uncertainty associated with laser-based measurements can be largely reduced compared to monochromator-based setups. The measurement of the laser wavelength can be realised by wavemeters with intrinsically low uncertainties. Furthermore, the high power and spectral purity of the tuneable laser radiation enable also other characterisations of radiometric detectors, such as the stray light and bandpass characterisation and corrections of compact and cost-efficient array spectroradiometers that are increasingly used in various detector and sensor applications using radiometric techniques.

\section{Tunable laser facilities at PTB}

At the Physikalisch-Technische Bundesanstalt (PTB) - the national metrology institute of Germany - investigations on narrow-band wavelength-tuneable continuous-wave (cw) laser systems used for absolute radiometry over limited spectral ranges started more than twenty years ago. The TULIP setup (Tuneable Lasers in Photometry) covering the broad spectral range from about $360 \mathrm{~nm}$ to $1000 \mathrm{~nm}$ [2] has now been upgraded with a tuneable quasi-cw femtosecond laser system covering the entire spectral range between $230 \mathrm{~nm}$ and $3000 \mathrm{~nm}$, which is sufficient for calibration of most radiometric detectors. This new system provides a much higher output power throughout the whole useable spectral range than the cw-lasers in use. In addition, a second laser facility PLACOS (P

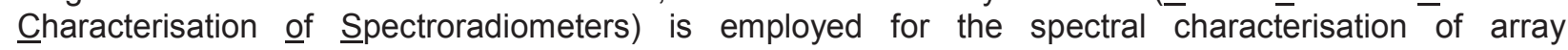
spectroradiometers throughout the spectral range from $220 \mathrm{~nm}$ to $2200 \mathrm{~nm}$ (see Fig. 1) [3]. The optical output power of the laser systems which are directly accessible without extensive re-adjustments of the setup is shown in Fig $1 \mathrm{~b}$.

The tuneable cW-lasers within the TULIP setup are pumped at $532 \mathrm{~nm}$ using a frequency doubled $\mathrm{Nd}: \mathrm{YVO}_{4}$ laser. Wavelength-tuneable radiation is provided by two dye lasers covering the spectral range from $565 \mathrm{~nm}$ to $700 \mathrm{~nm}$. A Ti:Sa laser with an additional intra cavity frequency doubling enables measurements over spectral ranges from $690 \mathrm{~nm}$ to $960 \mathrm{~nm}$ and $360 \mathrm{~nm}$ to about $460 \mathrm{~nm}$, respectively. The gap between $460 \mathrm{~nm}$ and $565 \mathrm{~nm}$ is partly covered by single $\mathrm{Ar}^{+}$- laser lines and the pump laser itself (not shown in Figure 1b). In the recently installed quasi-cw (pulse repetition rate $80 \mathrm{MHz}$ ) laser system, the spectral ranges from $680 \mathrm{~nm}$ to $1080 \mathrm{~nm}, 345 \mathrm{~nm}$ to $540 \mathrm{~nm}$ and $230 \mathrm{~nm}$ to $346 \mathrm{~nm}$ are covered by a quasi-cw mode-locked Ti:Sa laser and its frequency doubling and tripling, respectively. The spectral range above $1000 \mathrm{~nm}$ is covered by an optical parametric oscillator (OPO) with a tuneable signal range from $1000 \mathrm{~nm}$ to $1600 \mathrm{~nm}$. The useable range of the idler is from $1700 \mathrm{~nm}$ to $3000 \mathrm{~nm}$. Finally, the 
spectral range from $525 \mathrm{~nm}$ to $720 \mathrm{~nm}$ is covered by frequency doubling of the OPO signal. The pulse duration of the laser system varies between $130 \mathrm{fs}$ and $200 \mathrm{fs}$ but can be stretched up to DC using external components like integrating spheres.

The wavelength and the bandpass of the laser radiation are monitored continuously during the measurements. A calibrated wavemeter is used to measure the wavelength of the cw-lasers. A highresolution Echelle spectrograph calibrated by the cw lasers is applied to record the spectral distribution of the quasi-cw laser radiation. Due to the broadband nature of the femto-second pulses $(\sim 5 \mathrm{~nm}$ FWHM at $700 \mathrm{~nm}$ ) additional optical parts in the calibration setup are also needed in order to reduce the spectral bandwidth if required.

A uniform irradiance field, necessary for the measurement of large-area detectors is achieved by feeding the laser beams into either a $\mathrm{Ba}_{2} \mathrm{SO}_{4}$-covered integrating sphere via liquid light guides or into a tapered multimode fibre (TMF). Although producing the most uniform radiant field at the measurement plane, the integrating sphere is used only in the wavelength range above about $500 \mathrm{~nm}$ because of the well known fluorescence effects $[10,11]$. The use of TMF is the much higher throughput compared to an integrating sphere. The laser radiation also produces speckle patterns at the measurement plane due to its coherence properties which might affect the measurement result as well. Those undesired speckle effects are effectively suppressed by scrambling the propagation modes in the fibre by an ultrasonic bath and by an appropriate vibration unit attached to the liquid light guides [5].

A laser power stabiliser is used for active compensation of the drift and fluctuations of the lasers radiation. In addition, monitor technique is applied, where carefully positioned monitor detectors are installed to account for source dependent residual fluctuations and changes of the radiation field at the measurement plane during the measurement run.

As shown in Figure 1, the devices under test (DUT) as well as the reference detector are mounted on a three-axis translation stage located in a light-tight measurement chamber. Temperaturestabilised three-element reflection-type silicon trap detectors with precision apertures are used as reference standards, where the spectral power responsivity of the trap detectors is traceable calibrated with respect to the primary radiometric standard of PTB, a cryogenic radiometer [4].

The PLACOS facility uses an optical parametric oscillator (OPO) and a second-harmonic generator (SHG) [3]. The pump radiation for the OPO crystals is delivered by the $3^{\text {rd }}$ harmonic of a $\mathrm{Nd}$ :YAG laser $(355 \mathrm{~nm})$ operating at a repetition rate of $20 \mathrm{~Hz}$, where a pulse duration of about $5 \mathrm{~ns}$ is used. The resulting signal and idler waves of the OPO covers the spectral range from approximately $410 \mathrm{~nm}$ up to $2200 \mathrm{~nm}$. The SHG unit enables an extended range down to $220 \mathrm{~nm}$. In order to eliminate the laser radiation in the output beam at other than the selected wavelength, a set of specially designed dichroitic dielectric mirrors is employed. The power of the laser beam is adjusted as needed for the specific measurement by a variable neutral-density filter. The input optics of the spectroradiometer under test is placed in a light-tight enclosure so that the only radiation received is that of the laser. Before irradiating the input optics, the laser beam is homogenised using light shaping diffusers manufactured by holographic recording of randomised structures on quartz substrates.

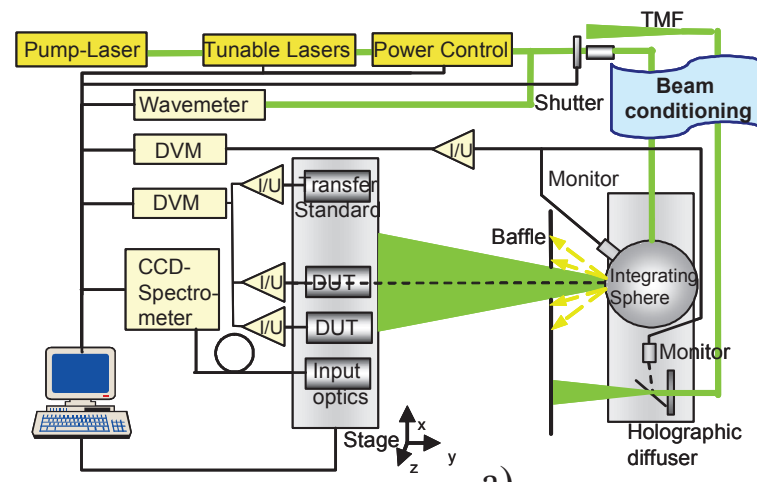

a)

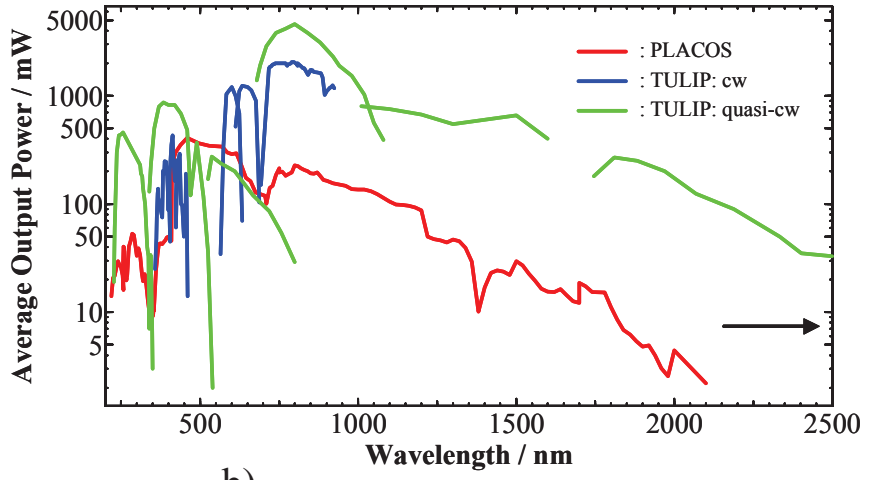

b)

Figure 1. a) Schematic representation of the tuneable laser facilities at PTB for calibration and characterisation of large area photometric and radiometric detectors as well as for characterisation of array spectroradiometers in a uniform radiation fields; b) Output power as a function of set wavelengths of the tuneable lasers used in TULIP and PLACOS setups. 


\section{Laser-based radiometric calibrations}

To demonstrate the advantages of the laser-based measurement approach, results of two types of measurements carried out at the TULIP facility are shown: the spectral irradiance responsivity of filtered detectors with diffusers and the radiance responsivity of a radiometer without a diffuser. The main difference in calibration of a detector with and without diffusers as entrance windows for radiation is that in the latter case interference effects are typically observed in the measurement data which need a proper handling. The spectral characterisation of large area detectors also requires uniform fields of high irradiance, which is the case in the TULIP setup.

Fig. 2a shows the performance of the TULIP setup for the spectral irradiance responsivity determination of three different photometers with diffusers, which are designed to match the spectral luminous efficiency function $V(\lambda)$ of the human eye. The photometers are different in terms of the size of the active area and the type of the filters used. Photometer No. 1 and No. 3 uses glass filters by means of a full filtering. Photometer No. 2 is matched to the $V(\lambda)$ function by using a mosaic glass filter. The spatial response uniformity of such a photometer is strongly varying with wavelength and, hence, the uniformity of the field is of critical importance for the results. The associated relative expanded uncertainties with a coverage factor of $k=2$ are shown in Fig. 2b.

The uncertainties of the measured responsivity values, as provided in Fig. 2b, were obtained using the Monte-Carlo simulation method [6]. The measurement uncertainties around the peak region of the responsivity curve are dominated by those uncertainty component caused by the calibration of the reference trap detector. Due to the filter characteristic of photometers, signals measured in the long and short wavelength range of the $V(\lambda)$ curve are very noisy, making the respective uncertainty component a dominant one.

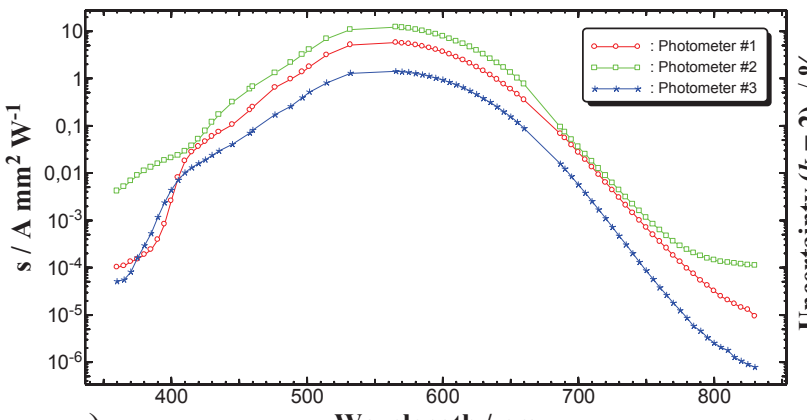

a)

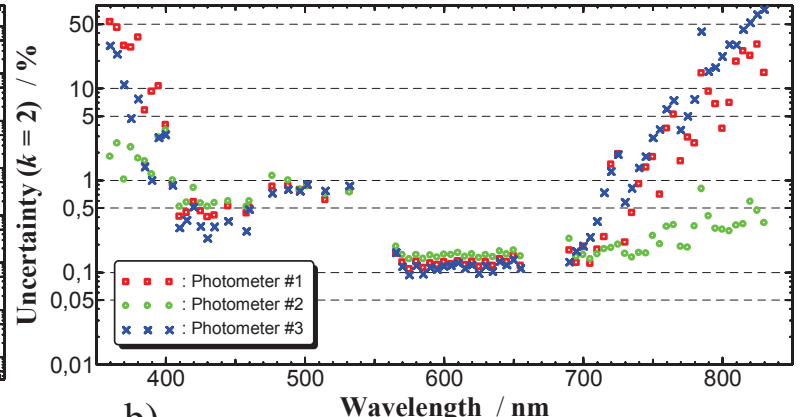

b)

Fig. 2. a) Spectral irradiance responsivity of three photometers with the diameter of the active area of \#1: $30 \mathrm{~mm}$, \#2: $10 \mathrm{~mm}$ and \#3: $30 \mathrm{~mm}$ measured with the cw-lasers in the TULIP facility; b) relative expanded uncertainties $(k=2)$ of the spectral irradiance responsivity values of photometer \#1 measured at the TULIP facility. The values are shown on a logarithmic scale.

To determine the radiance responsivity of a filtered detector without diffuser at a laser-based setup means sampling the filter function as detailed as possible including all interference fringes cause by the narrow-band coherent radiation. This is due to multiple reflections between the optical surfaces of the optic of a radiance detectors. As an example, the radiance responsivity of a pyrometer of type LP3 was measured at wavelength steps of $0.01 \mathrm{~nm}$ in the wavelength range between $620 \mathrm{~nm}$ and $680 \mathrm{~nm}$. The maximum allowed wavelength step size according to Nyquist criterion for this detector was found to be $0.03 \mathrm{~nm}$. The measured radiance responsivity with the interference oscillations and the associated measurement uncertainty are plotted in Fig. 3. The uncertainties are obtained using Monte-Carlo method. The measured data can be corrected for the interference oscillation using numerical calculations. The result is shown as blue curve in figure 3 . The uncertainty component resulting from the numerical algorithm has been considered in the uncertainty analysis. 
The broader bandpass of the monochromator causes averaging over the interference fringes. Therefore, compared to measurements with monochromator-based facilities, the measurements at the laser-based facility are more time consuming because the interference structures have to be sampled correctly. But due to the much lower signal-to-noise ratio of monochromator measurements the responsivity curve can only be determined over about four orders of magnitude, whereas the laser-based setup it able to determine responsivities over six orders of magnitude. This is especially important for pyrometers, where the integral out-of-band response of a filter radiometer has to be determined precisely. Also, the resulting measurement uncertainty determined for the laser-based measurement is smaller than that for monochromator-based measurements.

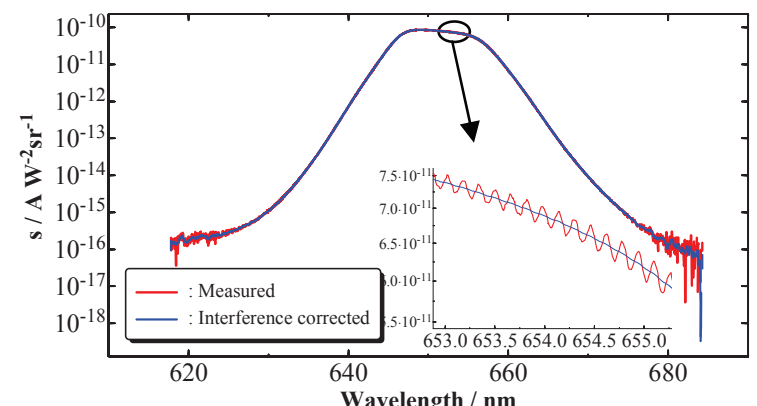

a)

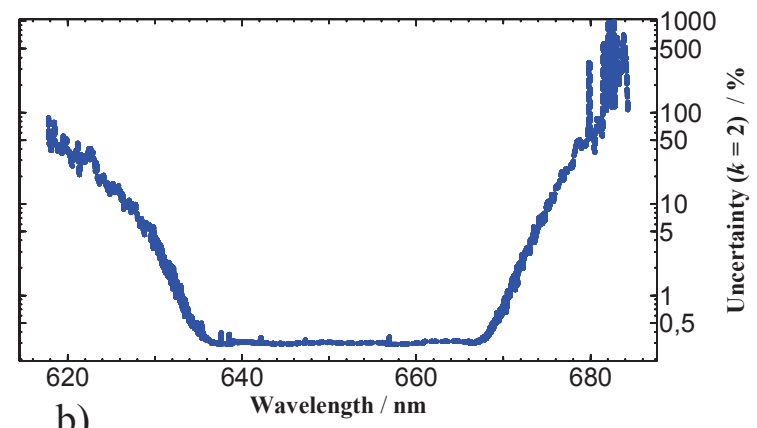

b)

Figure 3. a) Measured spectral radiance responsivity of a pyrometer (a filtered radiance detector). The close-up of the bandpass region shows interference fringes caused by the coherence of the laser radiation and the corrected data; $b$ ) relative expanded uncertainty $(k=2)$ of the spectral radiance responsivity of the pyrometer.

\section{Spectral characterisation and correction of array spectroradiometers}

Compact, cost efficient, fast and easy to use array spectroradiometers are increasingly used in various applications. Such devices, however, typically have a number of weak points. Such as a poor spectral stray light rejection (high out-of-band signal) that in general is considerably lower than that of a scanning double- or even single-grating monochromator systems. As a typically design feature the bandwidth of the devices as well as the slit (bandpass) function is varying throughout the spectral range.

With the help of the PLACOS setup, the response of array spectroradiometers to monochromatic excitation - the line spread function (LSF) - can be measured for any excitation wavelength throughout the spectral range from $220 \mathrm{~nm}$ to $2200 \mathrm{~nm}$. It has to be noted that the measured LSF contains both the information about the specific excitation wavelength, the stray light distribution in the array spectroradiometer and also the bandpass function. So that in principle these information can be used to correct stray light and bandpass distorted measurements numerically by using the measurement data from the PLACOS setup. The wide spectral coverage and the fine tuning capability of the laser wavelengths is advantageous for the characterisation of spectroradiometers having sharp features in their LSF, such as higher-order diffraction peaks and internal interreflections. In such cases acquisition of the LSF over a fine grid of excitation wavelengths is required. Otherwise the interpolation of the LSF over a wide gap between the excitation wavelengths (stimulated pixels) could yield large interpolation errors. [7]

Fig. 4 shows the response of a high-end CCD spectroradiometer to various monochromatic laser lines at several representative wavelengths. As one can see from the Fig. 4, the LSF has typical interreflection caused shoulders to the left of the bandpass region. The stray light level of the device ranges from about one part in $10^{3}$ in the vicinity of the bandpass peak to a few parts in $10^{6}$ at the far end from the excitation wavelength. Also higher order diffraction peaks can be observed. The results of the spectral characterisation can be used to determine the spectral correction matrices for the array spectroradiometers. The matrices then can be applied to the raw data of the spectroradiometers, i.e. both during the calibration of the spectroradiometers against the spectral irradiance transfer standards as well as to the measurement data. 


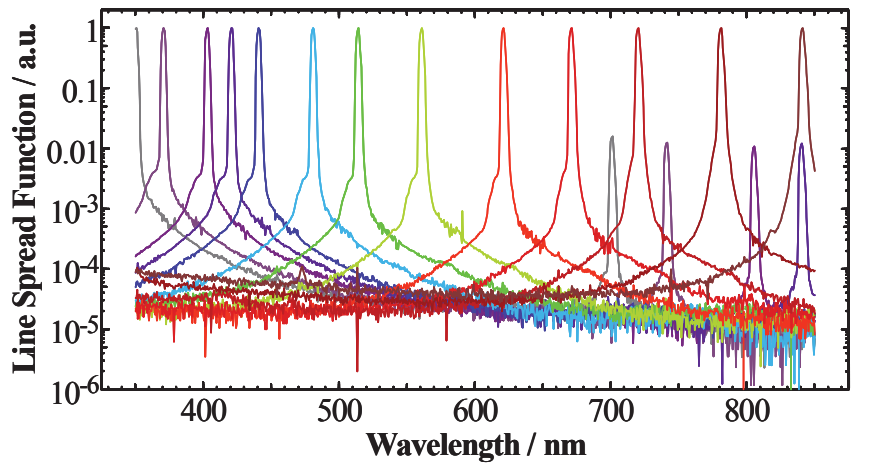

a)

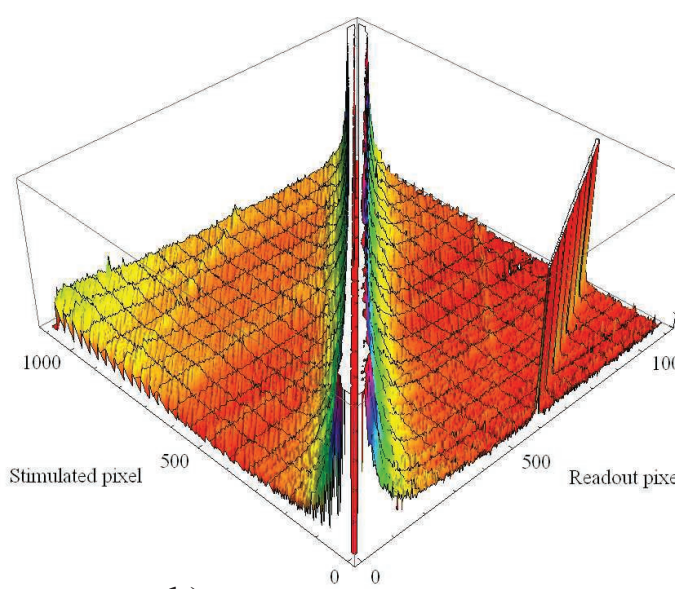

b)

Figure 4. a) Line spread function of a CCD array spectroradiometer at several wavelengths; b) 3D representation of stray light distribution matrix determined at the PLACOS setup. The second diffraction order and also interreflections can be seen clearly.

Figure 5 demonstrates the effectiveness of the spectral corrections applied to the spectral power distribution of high power LEDs measured by an array spectroradiometer. The bandpass and stray light correction of the LED measurement data is achieved as a product of the determined correction matrix and the data vector (for more detail see [8]).
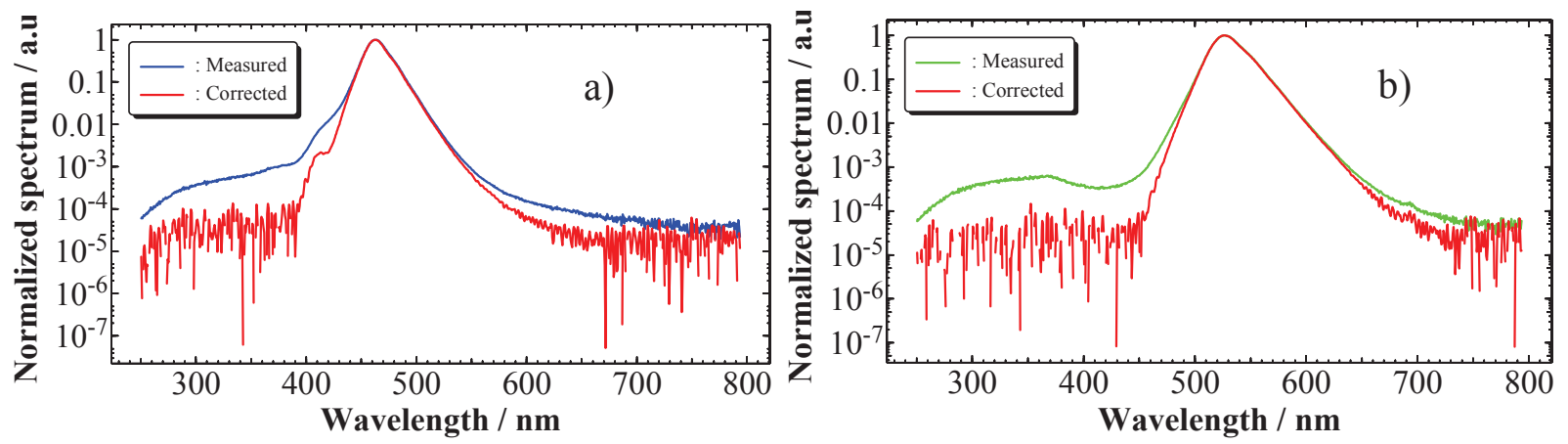

Figure 5. Spectral power distribution of a blue (a) and a green (b) high-power LED measured by a CCD-array spectroradiometer and the spectrally corrected data using the determined instrument matrix of the device.

\section{Summary}

The laser-based TULIP and PLACOS facilities for calibration and characterisation of radiometric detectors and sensors are presented. The effect and handling of unwanted interference effects have been explained. It has been shown that the higher output power of the laser-based setup and the smaller bandwidth of the laser radiation result in an reduction of measurement uncertainty that is about one order of magnitude with respect to monochromator based measurement. The advantage of laser based measurements is that steep responsivity curves of detectors and sensors can be measured with much more detail and lower uncertainties. It has also be shown that the interference fringes caused by this narrowband radiation can be corrected numerically so that the overall measurement uncertainty is very low.

The results of the stray light characterisation of array spectroradiometers have been presented. With this technique the stray light and the bandpass of low cost spectroradiometers can be corrected very effectively.

\section{Acknowledgement}

We thank our colleagues from the working group "7.31 High Temperature Scale" of the Berlin Institute of PTB for supporting the measurements of the radiance meter. 


\section{References}

1. S. Winter, A. Sperling, "Uncertainty analysis of a Photometer Calibration at the DSR Setup of the PTB", Proc. $2^{\text {nd }}$ Expert Symposium on Measurement Uncertainty (Braunschweig 2006), pp. 139-142, CIE x029:2006, ISBN 39810021-4-8.

2. A. Sperling, O. Larionov, U. Grusemann, S. Winter, "Stray-light correction of array spectrometers using tuneable pulsed and cw lasers," Proceedings of the $9^{\text {th }}$ international conference on new developments and applications in optical radiometry (NEWRAD), p. 93, Davos, 2005, ISBN-10 3-033-00570-5.

3. S. Nevas, M. Lindemann, A. Sperling, A. Teuber and R. Maass, "Colorimetry of LEDs with Array Spectroradiometers", MAPAN - Journal of Metrology Society of India, Vol. 24, No. 3, 2009;pp 153-162.

4. L. Werner, J. Fischer, U. Johannsen and J. Hartmann, "Accurate determination of the spectral responsivity of silicon trap detectors between $238 \mathrm{~nm}$ and $1015 \mathrm{~nm}$ using a laser-based cryogenic radiometer," Metrologia 37, 279-284 (2000).

5. A. Sperling and S. Nevas, "High accuracy measurements of the spectral responsivity of large area detectors for high irradiances using laser radiation," Proc. of NEWRAD: $10^{\text {th }}$ International Conference on New Developments and Applications in Optical Radiometry (2008).

6. Evaluation of measurement data - Supplement 1 to the "Guide to the expression of uncertainty in measurement" - Propagation of distributions using a Monte Carlo method JCGM 101:2008.

7. S. Nevas et al., Spectral and colorimetric characterization of LEDs using spectroradiometers, Light and Lighting Conference with special emphasis on LEDs and Solid State Lighting, Budapest, 27-29, May, 2009, Hungary.

8. S. Nevas et. al., Practical method for bandpass and stray light correction of array-spectroradiometer data, CIE Expert Symposium on Spectral and Imaging Methods for Photometry and Radiometry, 2010, Bern, 30-31, August, 2010, Switzerland.

9. Zong Y., Brown S. W., Johnson B. C., Lykke K. R., Ohno Y., Simple spectral stray light correction method for array spectroradiometers, Appl. Opt. 45, 1111-1119, 2006.

10. R. D. Saunders and W. R. Ott, Spectral irradiance measurements: effect of uv-produced fluorescence in integrating spheres, Appl. Opt. 15, 827-828 (1976).

11. Ping-Shine Shaw and Zhigang Li, On the fluorescence from integrating spheres, Applied Optics, Vol. 47, Issue 21, pp. 3962-3967 (2008) 